\title{
What can Software Engineers Learn from Manufacturing to Improve Software Process and Product?
}

\author{
Norman SCHNEIDEWIND \\ Information Sciences, Naval Postgraduate School, Monterey, US \\ Email: ieeelife@yahoo.com
}

\begin{abstract}
The purpose of this paper is to provide the software engineer with tools from the field of manufacturing as an aid to improving software process and product quality. Process involves classical manufacturing methods, such as statistical quality control applied to product testing, which is designed to monitor and correct the process when the process yields product quality that fails to meet specifications. Product quality is measured by metrics, such as failure count occurring on software during testing. When the process and product quality are out of control, we show what remedial action to take to bring both the process and product under control. NASA Space Shuttle failure data are used to illustrate the process methods.
\end{abstract}

Keywords: manufacturing methods, software process, software product, product and process quality, Taguchi methods

\section{Introduction}

Based on "old" ideas from the field of manufacturing, we propose "new" ideas for software practitioners to consider for controlling the quality of software. Although there are obviously significant difference between hardware and software, there are design quality control processes that can be adapted from hardware and applied advantageously to software. Among these are the Taguchi manufacturing methods, statistical quality control charts (statistical quality control is the use of statistical methods to control quality [1]), and design of experiments. These methods assess whether a product manufacturing process is within statistical control. Statistical control means that product attributes like software failure occurrence is within specified control limits.

Every process has inherent variation that hampers accurate process prediction. In addition, interactions of people, machines, environment, and methods introduce noise into the system. You can control the inherent variation by identifying and controlling its cause, thereby bringing the process under statistical control [2]. While this is important, we should not get carried away by focusing exclusively on process. Most customers do not care about process. They are interested in product quality! Thus, when evaluating process improvement, it is crucial to measure it in terms of the product quality that is achieved. Thus, we explore methods, like Taguchi methods, that tie process to product. We apply these methods to NASA Space Shuttle software using actual failure data.
The use of the Shuttle as an example is appropriate because this is a CMMI Level 5 process that uses product quality measurements to improve the software development process [3]. It is a characteristic of processes at this level that the focus is on continually improving process performance through both incremental and innovative technological changes and improvements. At maturity level 5, processes are concerned with addressing statistical common causes of process variation and changing the process (for example, shifting the mean of the process performance) to improve process performance. This would be done at the same time as maintaining the likelihood of achieving the established quantitative processimprovement objectives. While some software engineers argue that a CMMI Level 5 process is not applicable to their software, we suggest that it is applicable to any software as an objective, with the benefit of evolving to a higher CMMI level.

Typical metrics that software engineers use for assessing software quality are defect, fault, and failure counts, complexity measures that measure the intricacies of the program code, and software reliability prediction models. The data to drive these methods are collected from defect reports, code inspections, and failure reports. The data are collected either by software engineers or automatically by using a variety of software measurement tools. These data are used in design and code inspections to remove defects and in testing to correct faults.

Since software engineers are typically not schooled in 
manufacturing systems, they are unaware of how manufacturing methods can be applied to improving the quality of software. Our objective is to show software researchers and practitioners how manufacturing methods can be applied to improving the quality of software.

\section{Relationship of Manufacturing to Software Development}

There is a greater intersection between manufacturing methods and software development than software practitioners realize. For example, software researchers and industry visionaries have long dreamed about a Leg0 block style of software development: building systems by assembling prefabricated, ready-to-use parts. Over the last several decades, we have seen the focus of software development shift from the generation of individual programs to the assembly of large number of components into systems or families of systems. The two central themes that emerged from this shift are components, the basic system building blocks, and architectures, the descriptions of how components are assembled into systems. Interest in components and architectures has gone beyond the research community in recent years. Their importance has increasingly been recognized in industry. Today, a business system can be so complex that major components must be developed separately. Nevertheless, these individually developed components must work together when the system is integrated. The need for a component-based strategy also arises from the increased flexibility that businesses demand of themselves and, by extension, of their software systems. To address the business needs, the software industry has been moving very rapidly in defining architecture standards and developing component technologies. An emerging trend in this movement has been the use of middleware, a layer of software that insulates application software from system software and other technical or proprietary aspects of underlying run-time environments. [4] Component-based software construction enhances quality by confining faults and failures to specific components so that the cause of the problem can be isolated and the faults removed.

Manufacturing is an important part of any software development process. In the simplest case, a single source code file must be compiled and linked with system libraries. More usually, a program will consist of multiple separately written components. A program of this kind can be manufactured by compiling each component in turn and then linking the resulting object modules. Software generators introduce more complexity into the manufacturing process. When using a generator, the programmer specifies the desired effect of a component and the generator produces the component implementation. Generators enable effective reuse of high-level designs and software architectures. They can embody state of-the-art implementation methods that can evolve without affecting what the programmer has to write. While the programmer can manually invoke compilers and linkers, it is much more desirable to automate the software manufacturing process. The main advantage of automation is that it ensures manufacturing steps are performed when required and that only necessary steps are performed. These benefits are particularly noticeable during maintenance of large programs, perhaps involving multiple developers. Automation also enables other parties to manufacture the software without any detailed knowledge of the process. [5] Component reuse is a boon to software reliability because once a component is debugged, it can be used repeatedly with assurance that it is reliable.

Another tool borrowed from manufacturing is configuration control [6]. At first blush, one may wonder how configuration control fits with software development. Actually, it is extremely important because software is subject to change throughout the life cycle in requirements, design, coding, testing, operation and maintenance. A dramatic illustration of the validity of this assertion is the software maintenance function. For example, no sooner is the software delivered to the customer than requests for changes are received by the developer. This is in addition to the changes caused by bug fixes after delivery. If configuration management is not employed, the process will become chaotic with neither customer nor developer knowing the state of the software and the status of the process.

\section{Arguments against Applying Manufacturing Methods to Software}

We note that not all software engineers subscribe to the idea that manufacturing methods can be applied to software. They claim that it is infeasible to measure software because unlike high volume manufacturing of hardware, with close tolerances, many software projects are low volume produced with a fuzzy process, where product tolerance has no meaning. [7] Actually, none of this is true. While component-based software development is not a high volume process, it produces products for use in a large number of applications (e.g., edit module in a word processor). As for process, we have cited the CMMI Level 5 process that can control the variation in product quality by feeding back deviations in product quality to the development process for the purpose of correction the process that led to undesired variance in product quality. While it is true that software cannot be manufactured to the precision of software, statistical quality control of defect count, confidence intervals of reliability predictions, numerical reliability specifications, and quality prediction accuracy measurement, are just a few of the quantitative measurements from the physical world that have been applied to software, for example, in 
the Space Shuttle [3]. Thus, we are convinced that it is appropriate to apply manufacturing methods to software development in the sections to follow.

\section{Taguchi Methods}

Taguchi [1] revolutionized the manufacturing process in Japan through cost savings, for example, at Toyota. He understood that all manufacturing processes are affected by outside influences, like noise. Taguchi developed methods of identifying those noise sources that have the greatest effects on product variability. His ideas have been adopted by successful manufacturers around the globe because they created superior production processes at much lower costs.

Taguchi speeded up product and process design by separating controllable from uncontrollable variables. By concentrating on the controllable variables, fewer experiments are needed to arrive at the best product design. For example, in software, one of the key variables that is controllable is quality. On the other hand, user expectations are largely uncontrollable from the developer's perspective. However, controlling quality to achieve positive results, would contribute to meeting users' expectations.

Since a good manufacturing process will be faithful to a product design, robustness must be designed into a pro- duct before manufacturing begins. According to Taguchi, if a product is designed to avoid failure in the field, then factory defects will be simultaneously reduced. This is one aspect of Taguchi Methods that is often misunderstood. There is no attempt to reduce variation, which is assumed to be inevitable, but there is a definite focus on reducing the effect of variation. "Noise" in processes will exist, but the effect can be minimized by designing a strong "signal" into a product [8].

To relate software to Taguchi methods, we use the following definitions:

\subsection{Definitions}

Observation i: the recording of cumulative failures during test or operational time $t$.

$\mathrm{Y}_{\mathrm{i}}$ : value of observation $\mathrm{i}$ : $\mathrm{i}^{\text {th }}$ value of actual or predicted cumulative software failures

$\mathrm{T}_{\mathrm{i}}$ : specified target value of cumulative software failures for observation $\mathrm{i}$ (i.e., desire $\mathrm{Y}_{\mathrm{i}} \leq \mathrm{T}_{\mathrm{i}}$ )

Values of $T_{i}$ are listed in Table 1. In order to not bias the analysis, the individual failure counts $d_{i}$ were assigned a uniformly distributed number between 0 and 4 . Then these numbers were summed obtain the cumulative failure target values T. Software engineers could choose values appropriate for their software.

Table 1. Shuttle OI3 Loss Functions and Signal to Noise Ratios

\begin{tabular}{|c|c|c|c|c|c|c|c|c|c|}
\hline & & & & & & & & Forecasted & Forecasted \\
\hline & & Actual & Actual & Random & Cumulative & Actual & Predicted & Actual & Predicted \\
\hline & Failure & Failure & Cum ulative & Failure Count & Failure & Loss & Loss & Loss & Loss \\
\hline Test & Time & Count & Failures & T arget & $\mathrm{T}$ arget & Function & Function & Function & Function \\
\hline $\mathrm{I}$ & $\mathrm{t}$ & $\mathrm{d}_{\mathrm{i}}$ & $\mathrm{Y}_{\mathrm{ia}}$ & $\mathrm{T}_{\mathrm{i}}:$ & $\mathrm{T}_{\mathrm{i}}$ & $\mathrm{LF}_{\mathrm{a}}$ & $\mathrm{LF}_{\mathrm{p}}$ & LFia & LFip \\
\hline 1 & 0.97 & 2 & 2 & 2 & 2 & 0 & 8.55 & 3.54 & -9.75 \\
\hline 2 & 1.20 & 0 & 2 & 0 & 2 & 0 & 4.62 & 3.73 & -8.51 \\
\hline 3 & 3.03 & 2 & 4 & 0 & 2 & 4 & 3.62 & 5.18 & 1.17 \\
\hline 4 & 3.07 & 2 & 6 & 2 & 4 & 4 & 0.00 & 5.21 & 1.34 \\
\hline 5 & 4.00 & 0 & 6 & 1 & 5 & 1 & 0.01 & 5.95 & 6.21 \\
\hline 6 & 4.23 & 0 & 6 & 3 & 8 & 4 & 7.31 & 6.14 & 7.42 \\
\hline 7 & 8.20 & 0 & 6 & 1 & 9 & 9 & 3.86 & 9.29 & 27.69 \\
\hline 8 & 9.63 & 1 & 7 & 0 & 9 & 4 & 3.16 & 10.44 & 34.85 \\
\hline 9 & 9.70 & 0 & 7 & 2 & 11 & 16 & 14.23 & 10.49 & 35.18 \\
\hline 10 & 9.77 & 4 & 11 & 1 & 12 & 1 & 22.71 & 10.54 & 35.51 \\
\hline 11 & 10.37 & 0 & 11 & 3 & 15 & 16 & 59.57 & 11.02 & 38.48 \\
\hline 12 & 12.13 & 1 & 12 & 0 & 15 & 9 & 58.23 & 12.42 & 47.15 \\
\hline 13 & 27.67 & 1 & 13 & 4 & 19 & 36 & 133.41 & 24.78 & 117.83 \\
\hline 14 & 35.93 & 1 & 14 & 2 & 21 & 49 & 183.61 & 31.36 & 151.43 \\
\hline 15 & 87.53 & 1 & 15 & 3 & 24 & 81 & 273.91 & 72.41 & 298.15 \\
\hline \multirow[t]{8}{*}{16} & 136.53 & 1 & 16 & 2 & 26 & 100 & 344.11 & 111.40 & 336.92 \\
\hline & 140.00 & & & & & & & 114.16 & 335.96 \\
\hline & 145.00 & & & & & & & 118.13 & 333.70 \\
\hline & 150.00 & & & & & & & 122.11 & 330.42 \\
\hline & 155.00 & & & & & & & 126.09 & 326.12 \\
\hline & 160.00 & & & & & & & 130.07 & 320.80 \\
\hline & days & & & & & $\mathrm{SN}_{\mathrm{T}}$ & $\mathrm{SN}_{\mathrm{T}}$ & $\mathrm{SN}_{\mathrm{T}}$ & $\mathrm{SN}_{\mathrm{T}}$ \\
\hline & & & & & & 1.5773 & 1.9060 & 1.8595 & 2.1514 \\
\hline
\end{tabular}


LF: loss function (variability of difference between actual cumulative failures and target values during testing and operation)

$\mathrm{SN}_{\mathrm{T}}$ : signal to noise ratio (proportional to the mean of the loss function)

n: number of observations (e.g., number of observations of $\mathrm{Y}_{\mathrm{i}}$ and $\mathrm{T}_{\mathrm{i}}$ )

Taguchi devised an equation -- called the loss function (LF) -- to quantify the decline of a customer's perceived value of a product as its quality declines $[9,10]$ in Equation (1). This equation is a squared error function that is used in the analysis of errors resulting from deviations from desired quality. LF tells managers how much product quality they are losing because of variability in their production process. He also computed his version of the signal to noise ratio that essentially produces the mean of the loss function $[9,10]$ in Equation (2). Note that in most applications, a high signal to noise ratio is desirable. However, in the Taguchi method this is not the case because Equation (2) only measures signal, and no noise. Thus, since Equation (2) represents the mean value of the difference between observed and target values, a high value of $\mathrm{SN}_{\mathrm{T}}$ is undesirable.

$$
\begin{gathered}
\mathrm{LF}=\left(\mathrm{Y}_{\mathrm{i}}-\mathrm{T}_{\mathrm{i}}\right)^{2}, \\
\mathrm{SN}_{\mathrm{T}}=10 \log _{10}\left(\frac{\sum_{i=1}^{n}\left(Y_{i}-T_{i}\right)^{2}}{n}\right)
\end{gathered}
$$

The development of the loss function and signal to noise ratio, using the Shuttle failure data, involves the following steps:

1) First, we note that cumulative failure data are appropriate for comparing actual failure of a software release against the predicted values [11].

2) Thus, for release OI3 of the Shuttle flight software, we used actual cumulative failure data $\mathrm{Y}_{\mathrm{ia}}$ to compute the actual loss function $\mathrm{LF}_{\mathrm{a}}$ in Equation (1).

3 ) Then we used this loss function to compute the $a c$ tual signal to noise ratio $\mathrm{SN}_{\mathrm{T}}$ in Equation (2).

4) Then we used the predicted cumulative failure data $\mathrm{Y}_{\mathrm{ip}}$ to obtain the predicted loss function $\mathrm{LF}_{\mathrm{p}}$ and used this loss function to compute the predicted signal to noise ratio. The predicted cumulative failure counts were obtained from another research project using the Schneidewind Software Reliability Model (SSRM) [12]. Note in Figure 1 that there is a good fit with the actual data with $\mathrm{R}^{2}=.9359$. Also note that the failure times are long because the Shuttle flight software is subjected to continuous testing over many years of operation.

5) Next, the actual failure data $Y_{\text {ia }}$ were fitted, as a function of failure time $t$, with the regression Equation (3).

6) Then predicted failure data $Y_{\text {ip }}$ were fitted, as a function of failure time $t$, in the regression Equation (4), which also has a good fit to the data in Figure 1 with $\mathrm{R}^{2}$ $=.9701$.

7) Finally, signal to noise ratios were computed using Equation (2) for each of the four cases.

$$
\begin{gathered}
\mathrm{LF}_{\mathrm{ia}}=0.7956 \mathrm{t}+2.7771 \\
\mathrm{LF}_{\mathrm{ip}}=-0.0204 \mathrm{t}^{2}+5.3622 \mathrm{t}-14.912
\end{gathered}
$$

The four loss functions -- two actual and two predicted - are shown in Table 1 and Figure 1, along with the four corresponding signal to noise ratios. Figure 1 and Equations (3) and (4) can be used by a software engineer to forecast the loss function beyond the range of the actual and predicted loss functions.

\subsection{Results of Applying Taguchi Methods to Shuttle Software}

In Figure 1 and Table 1, we see that the loss functions

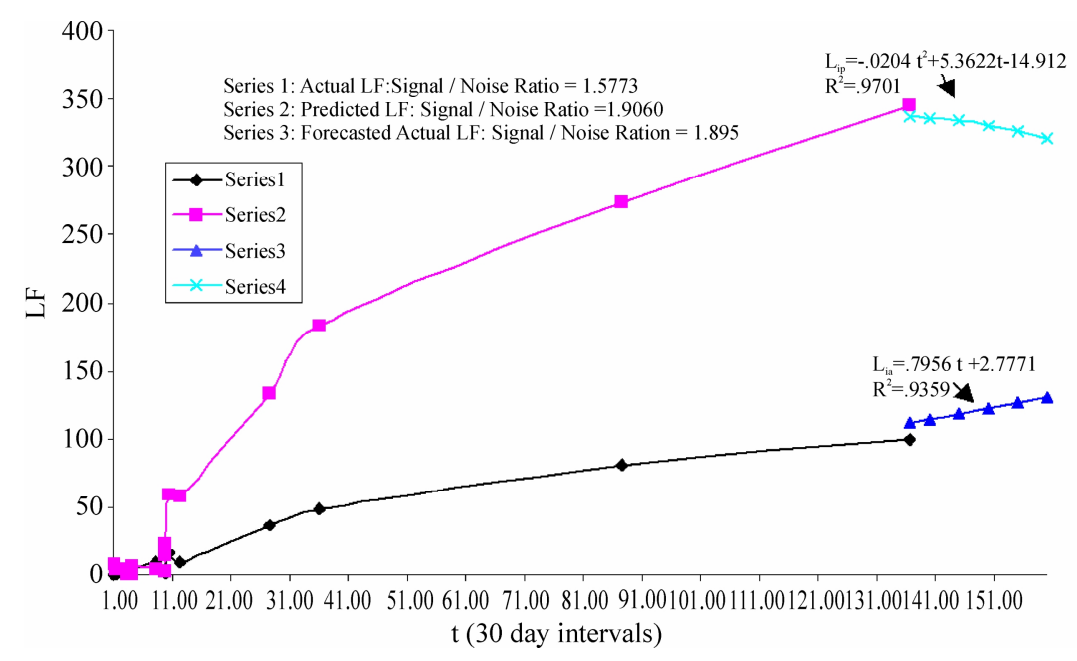

Figure 1. NASA space shuttle OI3: Loss function LF vs. failure time t 


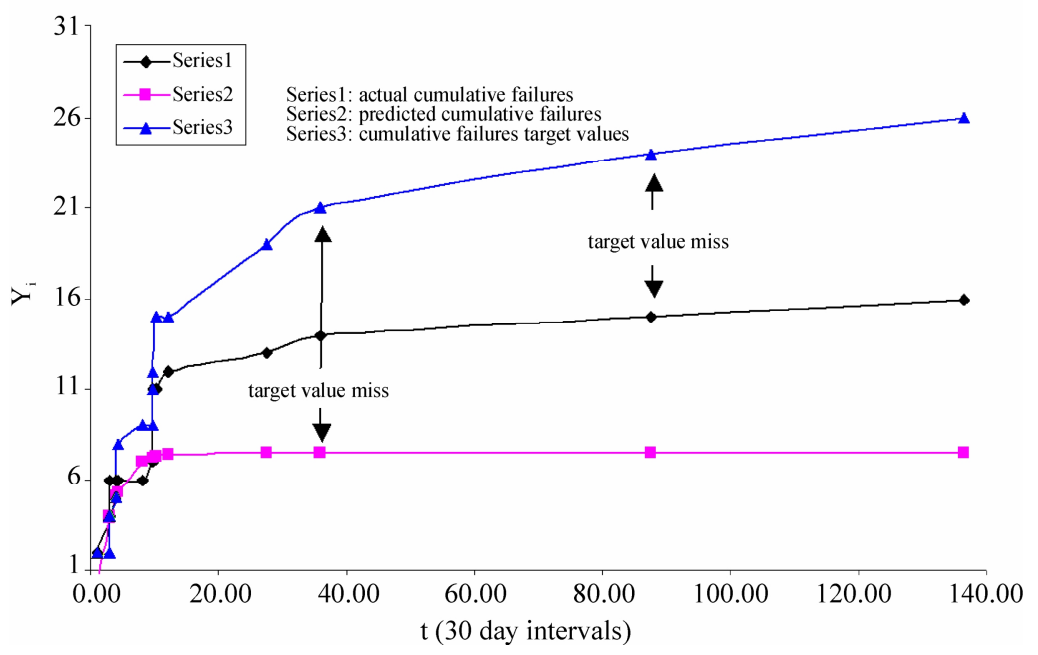

Figure 2. NASA shuttle OI3 cumulative failures $Y_{i}$ vs. failure time $t$

show that there is excessive variation between the desired and target values, based on both actual and predicted cumulative failures. Secondly, the mean variabilities, or signal to noise ratios, are large. This result suggests that the software production process requires tightened quality control.

Figure 1 and Table 1 reveal additional information about variability in loss functions, as given by the signal to noise ratio $\mathrm{S} / \mathrm{N}_{\mathrm{T}}$. We see that predicted values have greater variability than actual values and that forecasted values have the greatest variability of all LFs. The latter result is to be expected because forecasting into the unknown future is fraught with uncertainty. If the loss function could be reduced by exerting greater control over the software development process, the signal to noise ratio would also be reduced. By greater control we mean, for example, strengthening software inspection procedures and subjecting the software to stress tests to uncover and remove defects earlier in the development process.

Another perspective of applying Taguchi methods is shown in Figure 2, where we have plotted actual, predicted, and target cumulative failures against the time of failure for Shuttle release OI3. The message of this figure is that there is a large miss between the target values and the actual and predicted cumulative failures. Therefore, again, the remedy would be to strengthen the software development process by, for example, employing stringent inspection procedures to reduce the incidence of failures.

\section{Statistical Quality Control}

Now we illustrate another common manufacturing process control method that we show has applicability to software. Process control is based on two key assumptions, one of which is that random variability is basic to any production process. No matter how perfectly a proc- ess is designed, there will be some random variability, also called common causes, in quality characteristics from one unit to the next [13]. For example, a software development process cannot achieve perfection in specifying requirements, producing error-free code, inspecting and finding every defect, and finding and correcting every fault during testing. When undesirable variation in these activities becomes excessive, the process is out of control, and the search is on for assignable causes [13] For example, requirements analysts may be systematically misinterpreting customer requirements or software designers may make an incorrect mapping from requirements to code. In these situations, the process must be corrected.

Statistical quality control is based on the idea of monitoring quality and providing an alert when the process is out of control. Usually, variation is monitored and controlled by deviations from the mean, as computed by the standard deviation. Control is exercised by accepting the product if quality is within limits and rejected it, otherwise. For software, product metrics like the count of failures in excess of limits, as failures occur over a series of tests, can be used as a surrogate for identifying an out of control process.

As pointed out by [14], in hardware manufacturing, the number of observed failures is close to the actual number of failures that occur over time. In software, this is not the case. For example, a tester may observe two failures on a particular test, but the actual number of failures that would have occurred with a better test is ten. Thus, if the control limit were five, the tester would record this software as being under control on the control chart. To mitigate against this possibility, you should observe the trend of failure count, with increasing testing, to see whether the trend is increasing.

Statistical quality control for software uses the fol- 
lowing definitions and quality control relationships:

\subsection{Definitions}

n: Sample size

$\mathrm{d}_{\mathrm{i}}$ : failure count for test $\mathrm{i}$

$\overline{d_{i}}$ : Mean value of $d_{i}$

Sample $\mathrm{i}: \mathrm{i}^{\text {th }}$ observation of $\mathrm{d}_{\mathrm{i}}$ during test $\mathrm{i}$

$\mathrm{s}$ : Sample standard deviation of $\mathrm{d}_{\mathrm{i}}$

$\mathrm{d}_{\mathrm{i}}(\min )$ : minimum value of $\mathrm{d}_{\mathrm{i}}$

$\mathrm{d}_{\mathrm{i}}:(\max )$ maximum value of $\mathrm{d}_{\mathrm{i}}$

\subsection{Quality Control Relationships}

For the case where the standard deviation is used to compute limits, Equations (5) and (6) are used.

Upper Control Limit (UCL): $\overline{\mathrm{d}_{\mathrm{i}}}+\mathrm{Z} \mathrm{s}$

$$
\text { Lower Control Limit (LCL): } \overline{d_{i}}-\mathrm{Z} \mathrm{s}
$$

where $\mathrm{Z}$ is the number of standard deviations from the mean, which can be 1,2 , or 3 , depending on the degree of control exercised ( $Z=1$ : tight control, $Z=3$ : loose control).

\subsection{Results of Applying Statistical Quality Con- trol Methods to Shuttle Software}

Since the Shuttle is a safety critical system, we chose $\mathrm{Z}=$ 1 to provide tight control. Figure 3 reveals, as the Taguchi methods did, that there is an out of control situation when failure count become excessive -- in this case at test \# 10. In combining the results from Taguchi methods and statistical quality control, corrective action, like increased inspection, is necessary.

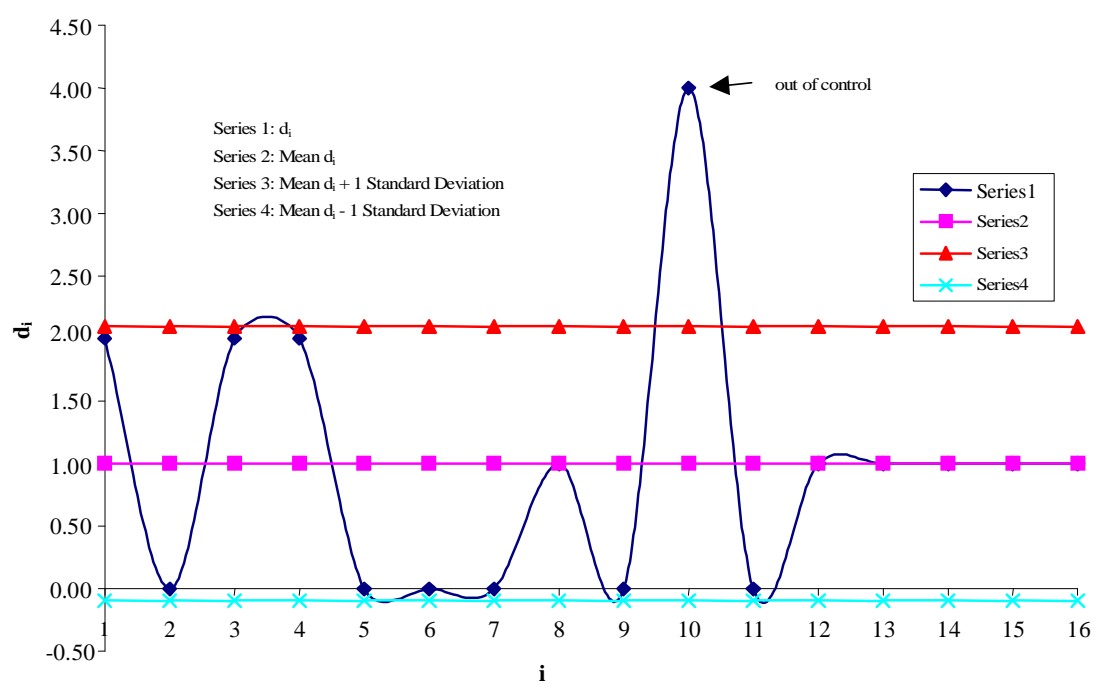

Figure 3. NASA space shuttle OI3: Failure count $d_{i}$ vs. test $i$

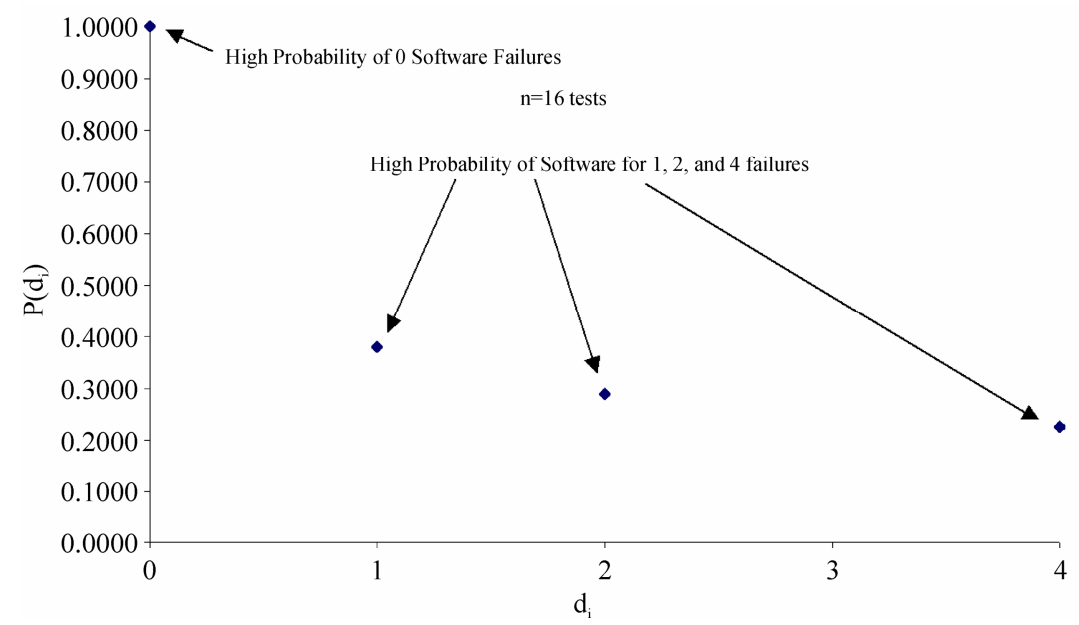

Figure 4. NASA space shuttle OI3: Probability of $d_{i}$ failures $P\left(d_{i}\right)$ on $n$ tests vs. $d_{i}$ 


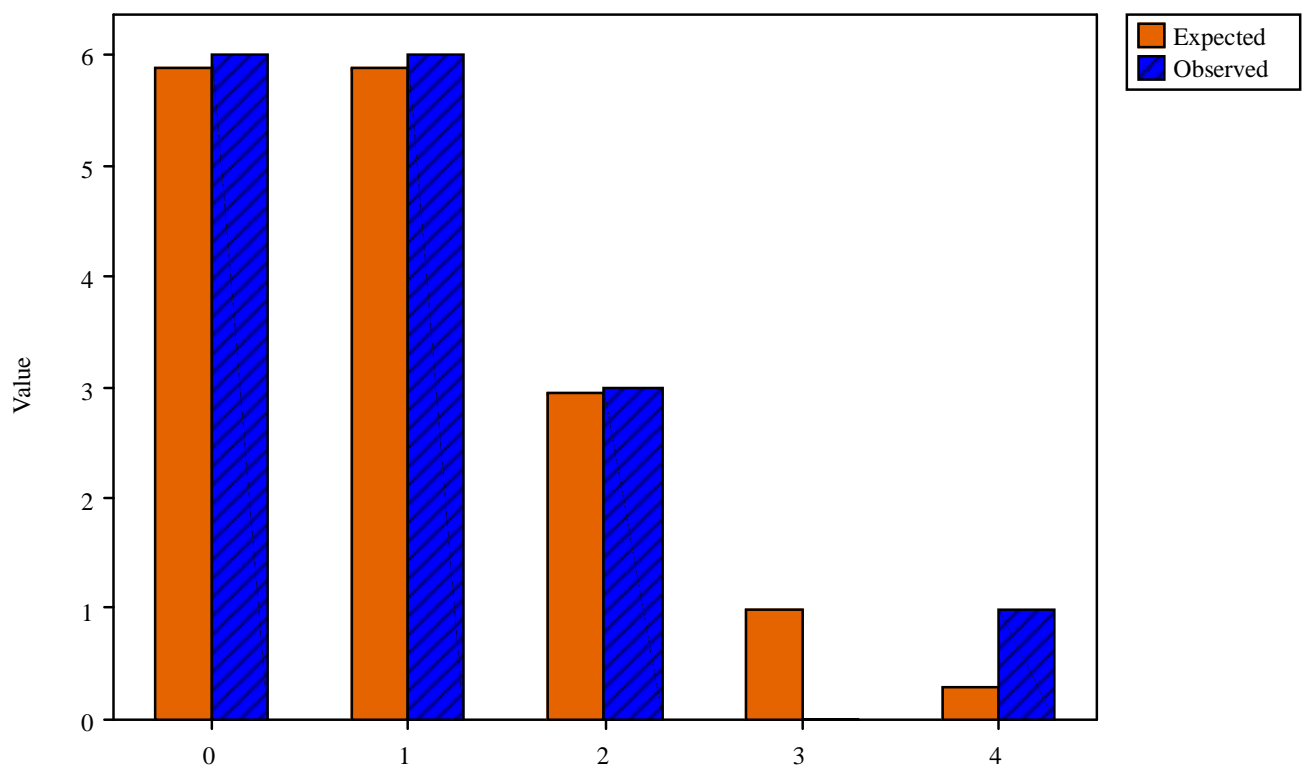

Figure 5. Test of poisson distribution of di number of failures during test $i$

\section{Statistical Process Control Using Failure Probability and Failure Counts [15]}

Next we show other methods of statistical process control that focuses on the probability of failures and number of failures occurring in a sample on a test.

\subsection{Probability of Failures during Tests}

We use probability of failure $\mathrm{P}\left(\mathrm{d}_{\mathrm{i}}\right)$ of $\mathrm{d}_{\mathrm{i}}$ failures during $n$ tests to control quality. This approach is based on the binomial distribution with probability $\mathrm{p}_{\mathrm{i}}$ of failures on test $i$ [16]. In our example, using the Shuttle data, the probability of failures $\mathrm{P}\left(\mathrm{d}_{\mathrm{i}}\right)$ is computed from the failure counts $d_{i}$ and $p_{i}$ in Equation (8). In order to compute $P\left(d_{i}\right)$, we first compute probability $\mathrm{p}_{\mathrm{i}}$ in Equation (7).

$$
\begin{gathered}
\mathrm{p}_{\mathrm{i}}=\mathrm{di} / \mathrm{n} \\
\mathrm{P}\left(\mathrm{d}_{\mathrm{i}}\right)=\left[\frac{\mathrm{n} !}{\mathrm{d}_{\mathrm{i}} !\left(\mathrm{n}-\mathrm{d}_{\mathrm{i}}\right) !}\right]\left[\mathrm{p}_{\mathrm{i}} \mathrm{d}_{\mathrm{i}}\right]_{\left[\left(1-\mathrm{p}_{\mathrm{i}}\right)^{\left.\mathrm{n}-\mathrm{d}_{\mathrm{i}}\right]}\right.}
\end{gathered}
$$

Applying Equation (8) to Figure 4, we note that there would be considerable risk in deploying this software in view of the high probability of failure for 2, 3, and 4 failures, given that 16 tests were used. An obvious remedy for this problem would be to remove the faults causing the failures before the software is deployed.

\subsection{Poisson Control Charts}

The Poisson control chart is based on the assumption that failure counts are distributed during tests according to a Poisson distribution. As can be seen in Figure 5, the observed failure counts $d_{i}$ on the $x$ axis are a good match with the expected counts for a Poisson distribution. Therefore, the Poisson distribution of failure counts, given in Equation (9), is used. There is sometimes a concern that the Poison distribution requires the assumption of independence of failures. Actually, dependence is not a frequent occurrence. For example, Musa [17] found that in 15 projects there was little association among failures.

$$
\mathrm{p}_{\mathrm{i}}=\frac{\overline{\mathrm{d}}^{\mathrm{d}} \mathrm{e}^{\mathrm{i}}}{\mathrm{d}_{\mathrm{i}} !}
$$

Next, having estimated $\mathrm{p}_{\mathrm{i}}$ in Equation (9), estimate the weighted mean number of failures across $\mathrm{n}$ samples, using Equation (10):

$$
\bar{P}=\sum_{i=1}^{n} d_{i} p_{i}
$$

Since the standard deviation s of the Poisson distribution is equal to square root of the mean, we have:

$$
\mathrm{s}=\sqrt{\bar{P}}
$$

Now, compute the lower control limit for the number of failures using Equations (10) and (11) and producing Equation (12):

$$
\mathrm{LCL}=\bar{P}-3 \mathrm{~s}
$$

It is necessary to compute the lower limit in order to identify quality that may be too high, resulting in waste of resources on software that does not require high quality.

Then, compute the upper control limit for the number of failures, again using Equations (10) and (11) and pro- 
ducing Equation (13):

$$
\mathrm{UCL}=\bar{P}+3 \mathrm{~s}
$$

Figure 6 confirms the result obtained in Figure 3 in that there is loss of control at test \# 10 where four failures occur. The faults causing these failures must be uncovered and removed.

\section{Design of Experiments [18]}

The last method in manufacturing process control we consider is the design of experiments that employs a hypothesis about the difference between desired (target) and actual values of a product attribute, such as cumulative failures, using software tests as the experiments. The idea is to use samples (software tests) and statistical tests to estimate whether there is a significant difference between desired and actual values of a product attribute. If the difference is significant, it may be attributed to process factors, such as deficient quality control. We apply this method to Shuttle software.

First determine whether the data are approximately normally distributed as required for using the $t$ test [16]. In Figure 7 it is shown that the cumulative failure data $Y_{i}$ are approximately normally distributed by virtue of the data (red) dots being close to the normal (blue) line.

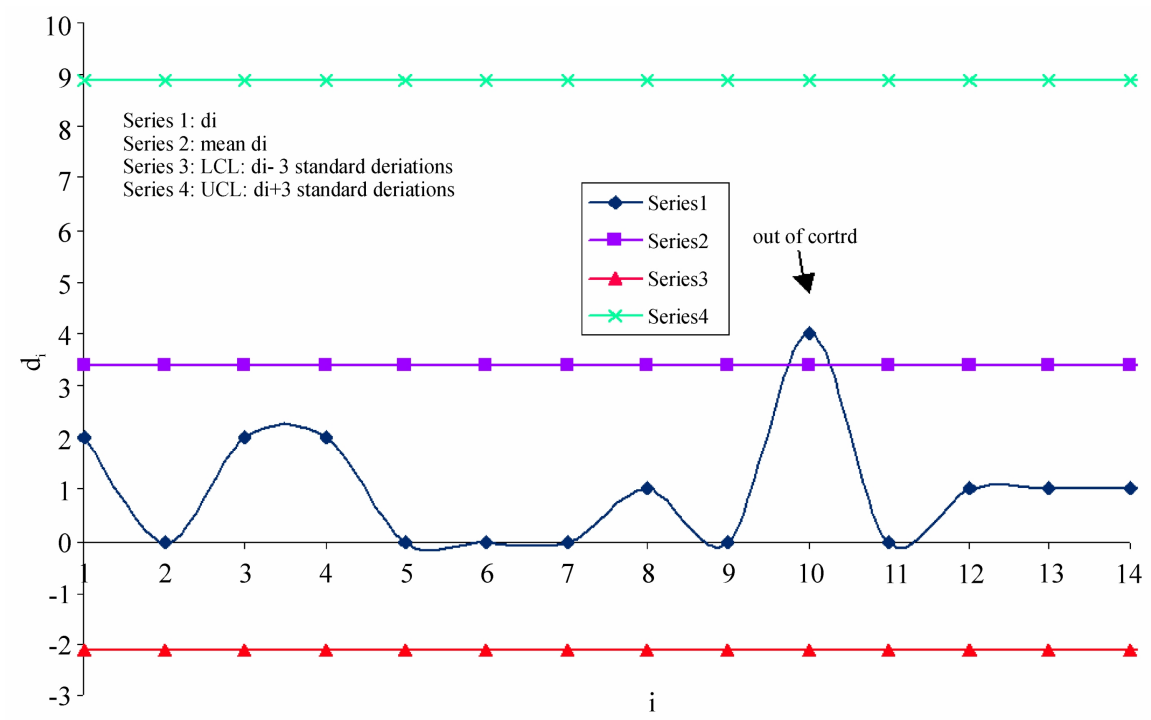

Figure 6. NASA space shuttle OI3; process control using poisson distribution: Number of failures $d_{i}$ vs. test number i

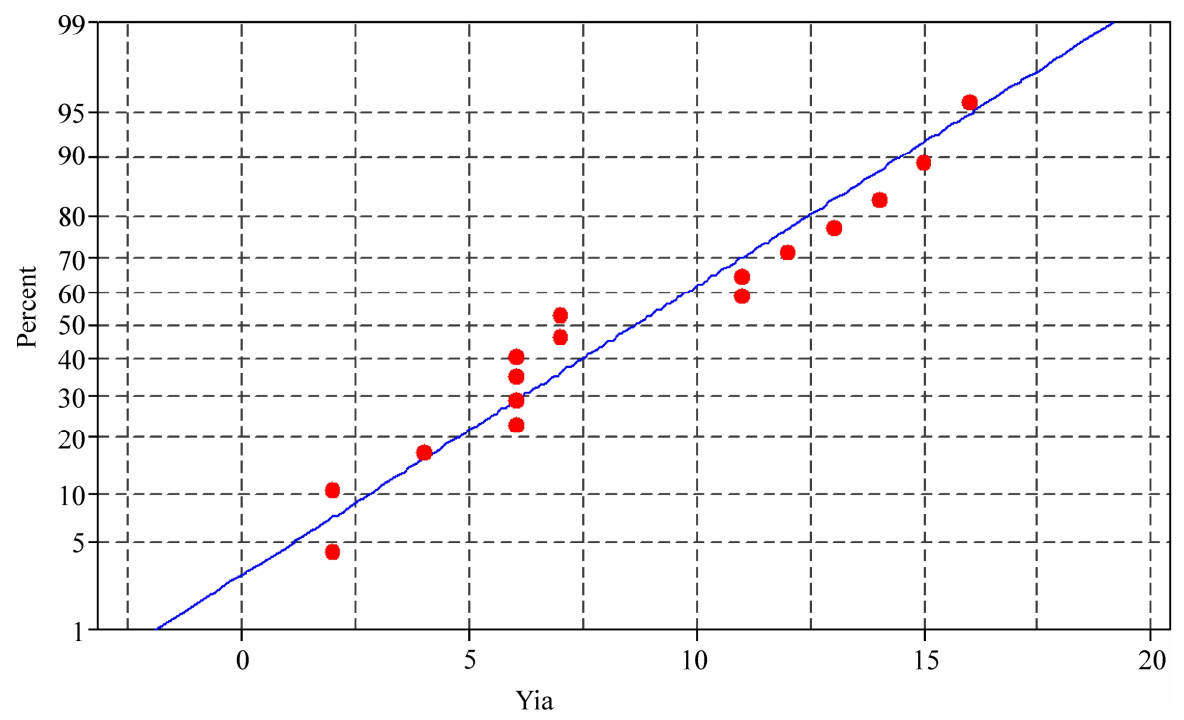

Figure 7. Normality test of cumulative number of failures yia 
Table 2. Shuttle OI3 design of experiments

\begin{tabular}{cccc}
\hline $\mathrm{T}_{\mathrm{i}}$ & $\mathrm{Y}_{\mathrm{ia}}$ & $\mathrm{s}$ & $\mathrm{t}$ \\
\hline 2 & 2 & & 1.7531 \\
2 & 4 & $\mathrm{\alpha}$ & $\mathrm{t}$ table \\
4 & 6 & $\mathrm{df}$ & 0.05 \\
5 & 6 & No statistically \\
8 & 6 & Significant difference \\
9 & 6 & & \\
9 & 7 & \\
11 & 7 & \\
12 & 11 & \\
15 & 11 & \\
19 & 13 & \\
21 & 14 & \\
24 & 15 & \\
26 & 16 & \\
11.5000 & 8.6250 & \\
$\overline{T_{i}}$ & $\overline{Y_{i}}$ & \\
7.8825 & 4.5295 & \\
$S_{T_{i}}$ & $S_{Y_{i}}$ & \\
\hline & & \\
\hline
\end{tabular}

Second, state the null $\mathrm{H}_{0}$ and alternate $\mathrm{H}_{1}$ hypotheses:

$\mathrm{H}_{0}$ : there is no statistically significant difference between $\mathrm{T}_{\mathrm{i}}$ and $\mathrm{Y}_{\mathrm{i}}$

$\mathrm{H}_{1}$ : there is a statistically significant difference between $\mathrm{T}_{\mathrm{i}}$ and $\mathrm{Y}_{\mathrm{i}}$

Third, compute the means of the cumulative failures and the target cumulative failures in Equations (14) and (15), respectively, and the standard deviation of the sum of the variance of $\mathrm{Y}_{\mathrm{i}}, \mathrm{s}_{\mathrm{Y}}{ }^{2}$, and variance of $\mathrm{T}_{\mathrm{i}}, \mathrm{s}_{\mathrm{T}}{ }^{2}$, in Equation (16)

$$
\begin{gathered}
\bar{Y}_{i}=\frac{\sum_{i=1}^{n} Y_{i}}{n} \\
\bar{T}_{i}=\frac{\sum_{i=1}^{n} T_{i}}{n} \\
\mathrm{~s}=\sqrt{{s_{Y_{i}}}^{2}+{s_{T_{i}}}^{2}}
\end{gathered}
$$

Fourth, based on the outcome of these computations, the $t$ statistic is computed in Equation (17).

$$
\mathrm{t}=\frac{\left(\bar{Y}_{i}-\bar{T}_{i}\right)}{s}
$$

Fifth, a comparison is made between the value computed from Equation (17) and the value obtained from the $t$ statistic table. If ( $\mathrm{t}<$ table value), accept $\mathrm{H}_{0}$ and conclude that the difference between the target and actual cumulative failures is not statistically significant; otherwise, accept $\mathrm{H}_{1}$ and conclude there is a difference, and attempt to assign the cause and correct the problem (e.g., inadequate software testing).

Based on Table 2, we accept $\mathrm{H}_{0}$ and conclude there is not a statistically significant difference between cumulative failures and the target values, based on the computed $\mathrm{t}=1.2650<\mathrm{t}$ table $=1.7531$. Thus with an error of $\alpha$ $=.05$ of rejecting $\mathrm{H}_{0}$, when in fact it is true, we have confidence that quality based on cumulative failures meets its goal.

\section{Conclusions}

We have presented some process methods from the field of manufacturing with the objective that the methods will prove useful to software engineers. While software development has some unique characteristics, we can learn from other disciplines, where the methods have been applied extensively on an international scale as part of a total quality management plan. Such a plan recognizes that process and product are intimately related and that the objective of software process improvement should be to improve software product quality.

The loss function and signal to noise ratio inspired by Taguchi methods proved to be valuable techniques for identifying and reducing excessive variation in software 
quality. In addition, statistical process control provided a method for identifying the test when the incidence of software failures was out of control. This allows the software engineer to estimate the number of tests to conduct in order to determine whether the software product is under control. Finally, design of experiments methodology allowed us to conduct hypothesis tests to estimate whether software product metrics were achieving their goals.

\section{REFERENCES}

[1] J. G. Monks, "Operations management," Second Edition, McGraw-Hill, 1996.

[2] A. L. Jacob and S. K. Pillai, "Statistical process control to improve coding and code review," IEEE Software, Vol. 20, No. 3, pp. 50-55, May/June, 2003.

[3] T. Keller and N. F. Schneidewind, "A successful application of software reliability engineering for the NASA space shuttle," Software Reliability Engineering Case Studies, International Symposium on Software Reliability Engineering, Albuquerque, New Mexico, November 4, pp. 71-82, 1997.

[4] J. Q. Ning, "Component-based software engineering (CBSE)," 5th International Symposium on Assessment of Software Tools (SAST'97), p. 0034, 1997.

[5] A. Sloane and W. Waite, "Issues in automatic software manufacturing in the presence of generators," Australian Software Engineering Conference, p. 134, 1998.

[6] Y. L. Yang, M. Li, and Y. Y. Huang, "The use of configuration conception in software development," IEEE Pacific-Asia Workshop on Computational Intelligence and Industrial Application, Vol. 2, pp. 963-967, 2008.

[7] R. V. Binder, "Can a manufacturing quality model work for software?" IEEE Software, Vol. 14, No. 5, pp. 101102,105, September/October 1997.
[8] M. Eiklenborg, S. Ioannou, G. King II, and M. Vilcheck, "Taguchi methods for achieving quality," San Francisco State University, School of Engineering. http://userwww. sfsu.edu/ gtarakji/engr801/wordoc/taguchi.html.

[9] R. K. Roy, "Design of experiments using the taguchi approach: 16 steps to product and process improvement," John Wiley \& Sons, Inc., 2001.

[10] G. Taguchi, S. Chowdhury, and Y. Wu, "Taguchi's quality engineering handbook," John Wiley \& Sons, Inc., 2005.

[11] Handbook of Software Reliability Engineering, Edited by Michael R. Lyu, Published by IEEE Computer Society Press and McGraw-Hill Book Company, 1996.

[12] N. F. Schneidewind, "Reliability modeling for safety critical software," IEEE Transactions on Reliability, Vol. 46, No. 1, pp. 88-98, March 1997.

[13] http://highered.mcgraw-hill.com/sites/dl/free/0072498919 195884/sch98919_ch09.pdf.

[14] N. Eickelmann and A. Anant, "Statistical process control: What you don't measure can hurt you!" IEEE Software, Vol. 20, No. 2, pp. 49-51, March/April, 2003.

[15] W. C. Turner, J. H. Mize, and J. W. Nazemetz, "Introduction to industrial and systems engineering," Third Edition, Prentice Hall, 1993.

[16] D. M. Levine, P. P. Ramsey, and R. K. Smidt, "Applied statistics for engineers and scientists," Prentice-Hall, 2001.

[17] J. D. Musa, A. Iannino, and K. Okumoto, "Software reliability: Measurement, prediction, application," McGrawHill, 1987.

[18] N. F. Fenton and S. L. Pfleeger, "Software metrics: A rigorous \& practical approach," Second Edition, PWS Publishing Company, 1997. 